\title{
AN ANALOG RF GAP VOLTAGE REGULATION SYSTEM FOR THE ADVANCED PHOTON SOURCE STORAGE RING
}

\author{
D. Horan ${ }^{*}$ and E. Cherbak \\ Argonne National Laboratory, 9700 South Cass Avenue, Argonne, IL 60439 U.S.A.
}

\section{Abstract}

An analog if gap voltage regulation system has been designed and built at Argonne National Laboratory to maintain constant total storage ring if gap voltage, independent of beam loading and cavity tuning effects. The design uses feedback control of the klystron modanode voltage to vary the amount of if power fed to the storage ring cavities. The system consists of two independent feedback loops, each regulating the combined rf gap voltages of eight storage ring cavities by varying the output power of either one or two if stations, depending on the mode of operation. It provides full operator control and permissive logic to permit feedback control of the if system output power only if proper conditions are met. The feedback system uses envelopedetected cavity field probe outputs as the feedback signal. Two different methods of combining the individual field probe signals were used to generate a relative DC level representing one-half of the total storage ring If voltage, an envelope-detected vector sum of the field probe if signals, and the DC sum of individual field probe envelope detector outputs. The merits of both methods are discussed. The klystron high-voltage power supply (HVPS) units are fitted with an analog interface for external control of the mod-anode voltage level, using a four-quadrant analog multiplier to modulate the HVPS mod-anode voltage regulator set-point in response to feedback system commands.

\section{APS GAP VOLTAGE CONTROL REQUIREMENTS}

The APS utilizes a $7-\mathrm{GeV}$ storage ring to generate synchrotron light for material research. The ring is designed to store $300 \mathrm{~mA}$ and has been operated routinely at $102 \mathrm{~mA}$ maximum current to date. The storage ring uses 16 single-cell cavities, arranged in groups of four at discrete sectors, to generate 9.4 megavolts of total rf gap voltage. Four 1-MW if stations are used to supply power to the cavities, and a waveguide switching/combining system allows operation of the storage ring with any two or more of the four if stations simultaneously.

Because the maximum beam loading in the storage ring cavities will represent a coupling coefficient of approximately 4 , the amount of if power required to maintain 9.4 megavolts of total if gap voltage varies widely depending on the amount of stored current [1].

\footnotetext{
* Work supported by U.S. Department of Energy, Office of Basic Energy Sciences, under Contract No. W-31-109-ENG-38.

\# Email: horan @aps.anl.gov
}

The present method used to regulate total storage ring gap voltage is a "control law" software program, utilizing two separate software amplitude control loops. This system has worked well, but it can only sample the cavity field probe powers and make adjustments to the rf system output power at a rate no faster than $1 \mathrm{~Hz}$. This slow data acquisition and transmission rate has caused delays in reducing the output power of the if stations, resulting in if system trips. The trips occur when the storage ring beam is suddenly dumped or lost, resulting in an instantaneous increase in the if power dissipation of the rf cavities by an amount equal to the beam loading effect. This sudden increase in power can degrade cavity vacuum and cause damage to cavity components such as tuners and couplers. The analog automatic gain control (AGC) system was developed to provide fast and accurate control of the if system power output as a function of stored beam current intensity.

\section{ANALOG REGULATION SYSTEM OVERVIEW}

The analog gap voltage regulation system is a true DCcoupled feedback system for maintaining constant if gap voltage amplitude in the APS storage ring cavities (see Fig. 1). The system consists of two identical and independent amplitude-control feedback loops. Each loop regulates the combined gap voltage of eight storage ring cavities (a sector-pair) by making real-time adjustments of the if power into the cavities in response to cavity beam-loading effects. This maintains agreement between the combined envelope-detected field-probe powers and an operator-selected gap-voltage setpoint.

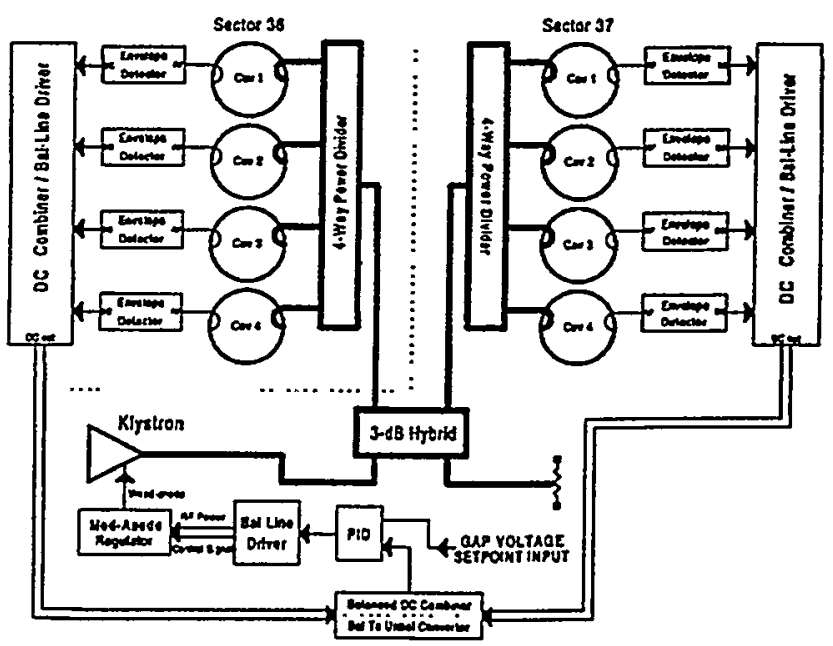

Figure 1: Analog rf gap voltage regulation system 


\section{DISCLAIMER}

This report was prepared as an account of work sponsored by an agency of the United States Government. Neither the United States Government nor any agency thereof, nor any of their employees, make any warranty, express or implied, or assumes any legal liability or responsibility for the accuracy, completeness, or usefulness of any information, apparatus, product, or process disclosed, or represents that its use would not infringe privately owned rights. Reference herein to any specific commercial product, process, or service by trade name, trademark, manufacturer, or otherwise does not necessarily constitute or imply its endorsement, recommendation, or favoring by the United States Government or any agency thereof. The views and opinions of authors expressed herein do not necessarily state or reflect those of the United States Government or any agency thereof. 


\section{DISCLAIMER}

Portions of this document may be illegible in electronic image products. Images are produced from the best available original document. 
At every sector, each of the four cavity field probes drives a dedicated envelope detector, the DC output of which is then converted from single-ended to. balanced output by an active line converter. The balanced outputs of all four balanced converters are then combined at each sector using a passive resistor network. This DC sum is sent to another DC-combiner chassis that sums the two sector-pair DC outputs together and generates a DC signal directly proportional to the combined field probe power in eight cavities. This signal is converted to single-ended topology and applied to the input of a VXI-based PID controller, which allows for remote control of proportional, integral, and differential (PID) gain in the feedback loop, plus remote loop setpoint adjustment. The PID controller output is then converted from single-ended to a pair of equal-amplitude balanced outputs, which are then each sent via single-pair cable to the external modanode voltage control input of the two rf stations that can drive the sector-pair of cavities. This signal controls the If system output power by increasing or decreasing the operator-selected klystron mod-anode voltage regulator setpoint value.

The AGC feedback system also includes control logic that permits feedback control of the If system output powers under conditional constraints. Each if station has a logic control chassis that allows feedback to be engaged on the if station only if all of the following conditions are met:

- system operator consciously selects gap voltage feedback operation,

- the collector-dissipation interlock is set [2],

- the particular If system output is switched into the ring,

- a minimum of seven out of the eight cavities in the sector-pair are in tune, and

- the klystron has a sufficient level of if drive.

If any of these conditions are not met, a situation is defined where feedback control of the rf system output power either is not desired or not possible. The control logic chassis will then disable the AGC feedback control within $6 \mathrm{~ms}$. This reaction time has proven fast enough to prevent klystron outgassing caused by sudden large increases in beam current when the AGC loop is suddenly opened.

\section{RF SYSTEM POWER OUTPUT CONTROL}

The AGC feedback system uses a four-quadrant analog multiplier [3] on an interface card to externally control the klystron mod-anode voltage (see Fig. 2). The DC control signal from the AGC feedback system is used as a value by which to multiply the operator-selected modanode regulator setpoint voltage, thus providing the ability to increase and decrease the klystron beam current in response to the feedback system commands. The external mod-anode control card converts the balanced-

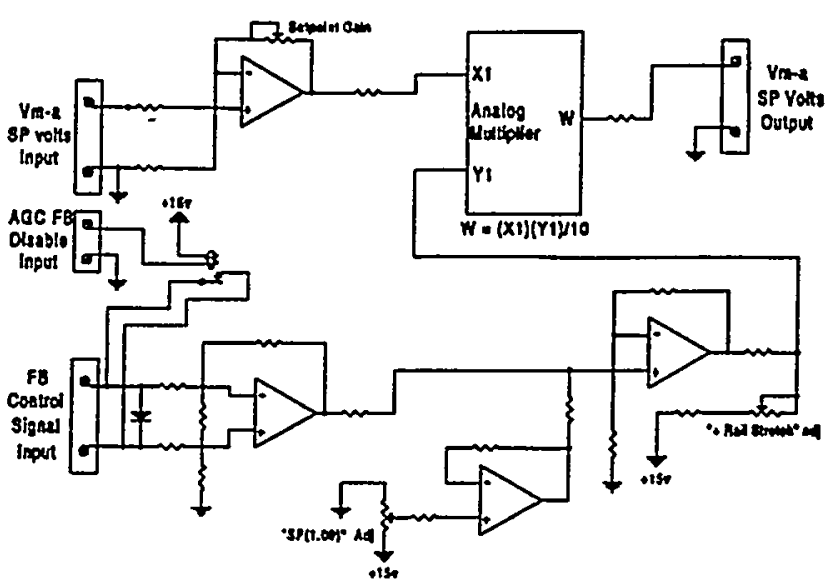

Figure 2: External mod-anode control interface

line feedback control signal to single-ended, and also diode-clips the feedback control signal in the negative direction so that the analog multiplier cannot generate a mod-anode setpoint voltage significantly lower than the operator-selected setpoint value. It provides DC offset controls to optimize the available range of the setpoint multiplication to a maximum of 1.5 , which will result in approximately $4 \mathrm{~dB}$ of klystron if output power control range. The use of an analog multiplier for this function has two discrete advantages, (1) the AGC feedback system can never increase the klystron mod-anode voltage by more than approx. 1.5 , and (2) the operator still has control of the baseline value for mod-anode setpoint. The feedback is disabled on the external control card by a fast reed relay that shorts the analog feedback control signal input when the feedback logic control chassis detects an operating condition that is not compatible with engaged feedback. The Experimental Physics Industrial Control System (EPICS) [4] remotecontrol screens have been developed to allow for remote operator control and monitoring of the feedback control logic chassis and system DC operating points from all operating locations.

\section{FEEDBACK SYSTEM PERFORMANCE}

Two methods of generating the analog feedback system input signal were tested to determine which would perform best for this application. One method used the vector-sum of eight field probe if signals (see Fig. 3), which was then envelope-detected to develop a DC voltage proportional to the amplitude of the combined field probe if signals. The other method used the DCsum of eight individual field-probe envelope detector outputs (see Fig. 1). The performance of both analog feedback system methods was measured. During these tests, eight storage ring cavities were under analogfeedback gap voltage control, while the other eight cavities were regulated using the traditional software control law gap-voltage regulation system. 


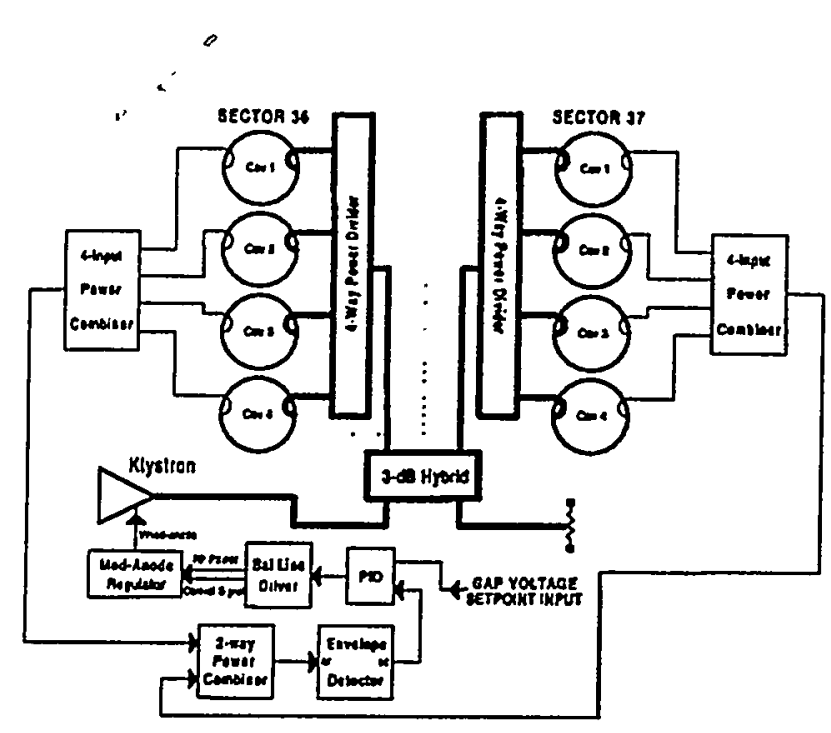

Figure 3: Rf vector-sum feedback input signal generation

It was found that using the vector-sum of eight fieldprobe rf signals as the feedback system input resulted in very poor gap voltage regulation in response to beam loading. At a stored current of $100 \mathrm{~mA}$, the combined gap voltage of the eight cavities under analog control had sagged approximately $14 \%$ from injection-power levels.

This poor regulation was caused by phase-to-amplitude cross-modulation of the field probe if signals when they were combined in the if regime. Because each of the storage ring cavities and their associated tuner control systems react differently to cavity beam loading effects, a vector-sum of individual cavity field probe signals will always contain an amplitude distortion generated by the differential phase shifts of the individual signals. This amplitude distortion affects the gap voltage regulation by allowing the beam to unevenly load the cavities without causing a corresponding reduction in the vector-sum field-probe signal applied to the gap voltage feedback system input. Phase-to-amplitude cross-modulation of the combined field-probe signals creates an artificial increase in total field probe signal level; therefore the feedback system does not call for more rf power to combat the beam loading effects, and the gap voltage sags as more beam is stored.

Using the DC-sum of individual field-probe envelope detector outputs as the feedback system input produced much better gap voltage regulation. By combining DC signals proportional to the amplitude of the individual cavity field probe powers, differential phase errors between the cavity field probe if signals are ignored; the resulting signal is a very sensitive indicator of the combined cavity $Q$ reduction caused by beam loading. It allowed the feedback system to accurately detect beam loading and increase the rf power to the cavities in order to maintain 9.4-MV total gap voltage, with no detectable sag from injection to $102 \mathrm{~mA}$ stored current.

Closed-loop frequency response measurements on the AGC feedback system were performed (see Fig. 4). The

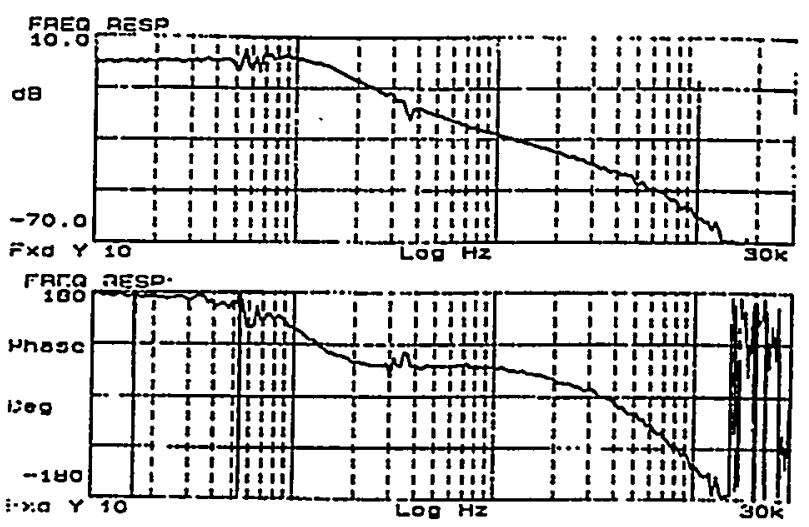

Figure 4: AGC system closed-loop response test

rapid roll-off of the loop frequency response above approximately $150 \mathrm{~Hz}$ is caused by the limited operating bandwidth of the mod-anode regulator.

\section{CONCLUSION}

The analog gap voltage regulation system has been successful in providing good storage ring gap voltage regulation in response to beam loading effects up to $102 \mathrm{~mA}$ stored beam. This system will reduce the number of klystron and cavity-related vacuum trips due to overpower events when beam is lost or dumped. Closedloop frequency response tests indicate that the loop operates in a stable region and has adequate bandwidth for the application. This system will be fully installed at APS by April of 1999.

\section{ACKNOWLEDGMENTS}

The authors would like to acknowledge Mike Phelan and Bill Yoder for their efforts in constructing and installing the feedback system hardware on schedule to meet studies deadlines.

\section{REFERENCES}

[1] Jin Wook Cho, Yoon Kang, "Measurement of Input Coupler Matching of a Loaded Storage Ring Single-Cell Cavity," Advanced Photon Source Light Source Note LS-247, August 14, 1995.

[2] The "collector interlock" is an interlock system that will limit klystron beam power if the if output of the klystron falls below a preset minimum for longer than 0.5 second.

[3] Analog Devices Engineering Staff, Nonlinear Circuits Handbook, Analog Devices, Inc., pp. 203-227 (1976).

[4] http://www.aps.anl.gov/asd/controls/epics/EpicsDocumentation 